\title{
Lower Collagen Area and Contralateral Lymph Node Metastasis Type Associated with Lower Disease- Free Survival in Thyroid Papillary Carcinoma
}

\section{Chengyun Dou}

the first affiliated hospital of uninversity of southern China

\section{Fen Tang}

the first affiliated hospital of university of southern China

Jianping Li

the first affiliated hospital of university of southern China

Qiang Zhao

the first affiliated hospital of university of southern China

Chuangjie Cao ( $\nabla$ caochj@mail2.sysu.edu.cn )

South China Normal University https://orcid.org/0000-0003-1423-6703

\section{Research}

Keywords: papillary thyroid carcinoma, collagen area, disease-free survival, lymph node metastasis

Posted Date: June 1st, 2020

DOl: https://doi.org/10.21203/rs.3.rs-31726/v1

License: (c) (i) This work is licensed under a Creative Commons Attribution 4.0 International License. Read Full License 


\section{Abstract}

Objectives: To introducing a novel prognostic risk scheme which based on collagen area and lymph node metastasis type in thyroid papillary carcinoma (PTC).

Method: Tumor collagen area and lymph node matastasis type as well as several other histomorphological factors with disease-free survival (DFS) were investigated in a corhort of 101 PTC patients.

Results: Median follow-up time of DFS was 76 months(inter quartile range: 71-83 months). Low collagen area and contralateral lymph node matastasis type were associated with dismal patient disease-free survival (DFS) significantly. While tumors with high collagen area showed a mean DFS of 77.37 months, gradually decreased to 76.53 months for tumors with moderate collagen area and to 66.67 months for tumors with low collagen area $(p=0.028)$. Negtive, central and ipsilateral lymph node metastasis showed mean DFS of $72.72,74.8$ and 72.81 months respectively, decreased to 62.17 months with bilateral lymph node metastasis and 59.43 months with contralateral lymph node metastasis $(p=0.029)$. In the cohort, uinivariate statistical analysis of the novel risk scheme revealed that the hazard ratio (HR) for DFS was 5.18 for R2 and 15.53 for R3 tumors compared to R1 PTC $(p=0.003)$. Disease free survival dropped from 77.74 months for R1 tumors to 68.7 months for R2 and 56.38 months for R3. Multivariate statistical analysis of the novel risk scheme revealed that the HR for DFS was 3.259 ( $95 \%$ confident interval [Cl] $1.122-9.469, p=0.03)$.

Conclusion: Our novel risk algorithm incorporating tumor collagen area and lymph node matastasis type allows strongly prognostic stratification of PTC. We suggest this risk algorithm as a morphology-based parameter for the routine diagnostic assessment of this tumor entity.

\section{Introduction}

The incidence of papillary thyroid carcinoma is increasing rapidly worldwide ${ }^{1}$. Disease recurrence rate ranges between $10 \%$ and $28 \%{ }^{2-4}$. Potential prognostic markers of PTC are aggressive histology, extrathyroidal extension, incomplete tumor resection, vascular invasion, multifocality, $>5$ involved lymph node $(0.2-0.3 \mathrm{~cm})$, lymph node metastasis $>3 \mathrm{~cm}$, distant metastases, and BRAF and TERT gene mutations ${ }^{5}$. Lymph node ratio in disease recurrence have prognostic significance in PTC as well ${ }^{6}$. However, these potential prognostic markers did not include accurate prognostic patient stratification according to morphological factors.

Guidelines of the WHO introduced a risk stratification system according to clinical outcomes following total thyroidectomy and radioiodine remnant ablation or adjuvant therapy ${ }^{5}$. Also, WHO-based grading of PTC does not allow prognostic patient stratification according to morphological factors.

To clarify this question, we evaluated three common morphological factors: tumor collogen area, lymphatic follicles around tumor and calcification, as well as other clinicopathological factors (age, 
gender, operative method, lymph node matastasis type and T stage). All factors regarding disease-free survival(DFS) were assessed.

\section{Patients}

The records of 222 patients treated surgically for PTC between 2012 and 2015 were identified from database of the first affiliated hospital of southern china. Of these, one hundred and three patients had pathologic confirmation and available follow-up information. Two patients died and were excluded from analysis. Finally, 101 patients were included in this study.

\section{Histological evaluation}

Full block haematoxylin and eosin stained PTC slides of every case were evaluated by two pathologists (CCJ, TF)who were blinded to clinicopathological data and follow-up. Furthermore, 10 randomly selected cases from the cohort were evaluated by ZQ consecutively in order to investigate interobserver reproducibility. According to tumor-node-metastasis staging system of the American Joint Committee on Cancer $^{4}$, regional lymph node metastasis is classified as central lymph node metastasis (N1a) and lateral lymph node metastasis (N1b). As was shown in table S1, we subdivided lymph node metastasis type into five subgroups (Table S1). Collagen types were divided into three categories according to the area of collagen and tumor area(Figure S1). Calcification condition were divided into two groups: present and absent of calcification. Lymphatic follicles around tumor were also evaluated: area with more than 5 lymphatic follicles /HPF around tumor were in one group; the rest were in the other group.

\section{Statistics}

Statistical analysis was performed using SPSS 20. Desease free survival probabilities were plotted with the Kaplan-Meier method, a log-rank test was used to probe for the significance of differences in survival probabilities. All significant parameters in univariate analysis were used in the multivariate model and excluded for $P>0.05$. Multivariate survival analysis was performed with the Cox proportional hazard model. $P$ values 0.05 were considered siginificant.

\section{Results}

\section{The factors related with disease-free survival}

101 PTC patients with operation were involved. The median age was 48 years old (inter quartile range: 37-55 years). Median follow-up time of disease-free survival (DFS) was 76 months(inter quartile range: 71-83 months). Collagen area was significantly associated with increased DFS in the test: while tumors with high collagen area showed a mean DFS of 77.37 months, gradually decreased to 76.53 months for tumors with moderate collagen area and to 66.67 months for tumors with low collagen area $(p=0.028$, 
Table S1 and Figure S2A). Lymph node metastasis type also showed prognostic for DFS: negtive, central and ipsilateral lymph node metastasis showed mean DFS of 72.72, 74.8 and 72.81 months respectively, decreased to 62.17 months with bilateral lymph node metastasis and 59.43 months with contralateral lymph node metastasis ( $p=0.029$, Table S1 and figure S2B). Other clinicopathological factors showed no association with DFS in the test(Table S1).

\section{Composition of the grading system}

We established a novel risk scheme based on collagen area and lymphnode metastasis type (Table 1), attributing a score to both collagen type (1-2 points) and lymphnode metastasis type(1-2 points). Tumors with moderate and high collagen area received a score of 1 and tumors with low collagen area had a score of 2. Likewise, negative metastasis in all surgical removed lymphnodes (negative), central and ipsilateral positive metastasis in surgical removed lymph nodes received 1 point, while bilateral and contralateral were scored 2 points. R1 (Low risk) tumors had a score of 2. Tumors had a score of 3 or 4 representing R2 (Moderate risk) or R3 (High risk) respectively. The composition of the risk scheme is summarized in Table 1. With R1 as a reference, DFS hazard ratio (HR) for G2 tumors was 5.18 and increased to 15.53 for $\mathrm{G} 3$ tumors ( $p=0.003$, table $S 2)$. 
Table 1

Algorithm for determining tumour risk from tumour collagen type and lymph node metastasis type

\section{Risk proposal for PTC}

\section{Collagen area}

Low

2

Moderate

1

High

1

\section{Lymph node metastasis type}

Negative 1

Central 1

Ipsilateral 1

Bilateral 2

Contralateral

2

Tumor risking total score

R1 2

$\mathrm{R} 2$ 3

R3

4

Low collagen area( $<20 \%$ of the whole tumor area); Moderate collagen area $(20-50 \%$ of the whole tumor area); High collagen area ( $>50 \%$ of the whole tumor area).

\section{Prognostic impact of the novel risk approach}

The novel risk approach had significant impact on DFS. Disease free survival dropped from 77.74 months for R1 tumors to 68.7 months for R2 and 56.38 months for R3 ( $p<0.001$, Figure S 2C). In multivariate analysis, the novel risk scheme revealed a significant independent prognostic impact for DSF $(p=0.03$, hazard ratio[HR] 3.259, 95\% confident interval [Cl] 1.122-9.469, table S3).

\section{Discussion}

Age and gender were reported as significant parameters in DFS for thyroid cancer in some studies ${ }^{8-10}$. In our study, age and gender did not significantly associated with DFS. The critical reason may be the heterogeneous conditions of other studies; including all the differentiated thyroid cancers in the study other than papillary thyroid carcinoma. 
American thyroid association (ATA) introduced a risk stratification system according to clinical outcomes following total thyroidectomy and radioiodine remnant ablation or adjuvant therapy. Intrathyroidal PTCs of all sizes were included in the ATA low-risk category, the risk of structural disease recurrence decreased from $8-10 \%$ in intrathyroidal PTC $>4 \mathrm{~cm}^{11}$, to $5-6 \%$ in $2-4 \mathrm{~cm}$ intrathyroidal PTC ${ }^{11}$, to $4-6 \%$ in multifocal papillary microcarcinomas and $1-2 \%$ in unifocal papillary microcarcinomas ${ }^{12,13}$. In our study, T stage classification not only included intrathyroidal PTCs, but also other parameters. Therefore, the significance of T stage in DFS may not be displayed, and this was in accordance with Yuksel UM et.al's study ${ }^{14}$.

A highly prognostic grading approache for uterine cervix ${ }^{15}$, pulmonary ${ }^{16}$, and several squamous cell carcinomas was based on tumor budding and cell nest size. We also assessed these histopathological parameters in PTC. However, interobserver reproducibility between pathologists were not high, and probably due to glands features. A prognostic morphological factor that associated with DFS was collagen area in PTCs. Moderate and high collagen area associated with higher DFS than low's $(p=$ 0.028). Zeng $R$ et al. reported that there is a positive assosiation between hashimoto's thyroiditis and PTC in children and adolescents. A prominent characteristic of hashimoto's thyroiditis is the presence of lymphatic follicles ${ }^{17}$. However, we did not found significant association between lymphatic follicles around tumor and DFS. Calcification could be found in PTC occassionally. Neither, this morphology feature showed association with survival in the cohort.

The prognostic significance of nodal metastases from papillary thyroid carcinoma can be stratified based on the size and number of metastatic lymph nodes, as well as the presence of extranodal extension ${ }^{18}$. With ipsilateral lymph node metastases, contralateral lymph node metastases are more prevalent ${ }^{19}$. However, it is not impossible that papillary thyroid carcinoma located in one lobe showing contralateral but not ipsilateral N1b. Based on N1a and N1b of American Joint Committee on Cancer, lympho node metastasis type were subdivided into 5 types in our study. Bilateral and contralateral lymph node metastasis type were significantly associated with shortened DFS $(p=0.029)$. This was inaccordance with Yuksel, U. M.'s study ${ }^{14}$. Two operative methods were included in this study: total thyroidectomy with lymph node dissection and subtotal thyroidectomy with lymph node dissection. This operative factor showed no association with DFS.

Knezević-Obad A et al. reported that positive cytologic examination played an important role in determing total thyroidectomy for patients ${ }^{20}$. To the best of our knowledge, no risk scheme could indicate a higher or lower DFS with collagen area morphological factor. Our risk scheme contained collagen area and metastasis type and showed as an independent prognostic factor for DFS in PTCs.

Our retrospective study is limited by the fact that our analyses contains a small sample size. The initial corhort included two patients who were died due to PTC recurrence. However, the sample was not enough for stastistical analysis. 
Taken together, the novel risk scheme contains collagen area and metastasis type and is easy to implement. Our data underlines the assumption that tumor collagen area and lymph node metastases type have the potential to constitute the pillars of a highly prognostic DFS risk scheme in PTC.

\section{Declarations}

\section{Ethics approval and consent to participate}

Ethical approval was approved by the EthicsCommittee of the First Affiliated Hospital of Universityof Southern China and informed consent wasobtained from all patients involved in this study

\section{Availability of data and material}

The data that support the findings of this study are available on request from the corresponding author.

\section{Conflict of interest}

The authors declare that they have no conflict of interest.

\section{Funding}

Funding information is not applicable.

\section{Authors' contributions}

TF collected data and evaluated slides. DCY data processing and statistical analysis. CCJ evaluated slides and wrote the paper. ZQ evaluated slides. LJP collected data.

\section{Consent for publication}

Consent for publication are received from all authors.

\section{References}

1. Davies, L. \& Welch, H.G. (2006) Increasing incidence of thyroid cancer in the United States, 19732002. JAMA 295, 2164-2167.

2. Kim, S.J., Park, S.Y., Lee, Y.J., Lee, E.K., Kim, S.K., Kim, T.H., Jung, Y.S., Ryu, J., Myong, J.P. \& Chung, K.W. (2014) Risk factors for recurrence after therapeutic lateral neck dissection for primary papillary thyroid cancer. Ann Surg Oncol 21, 1884-1890. 
3. Lee, C.W., Roh, J.L., Gong, G., Cho, K.J., Choi, S.H., Nam, S.Y. \& Kim, S.Y. (2015) Risk factors for recurrence of papillary thyroid carcinoma with clinically node-positive lateral neck. Ann Surg Oncol 22, 117-124.

4. Bardet, S., Malville, E., Rame, J.P., Babin, E., Samama, G., De Raucourt, D., Michels, J.J., Reznik, Y. \& Henry-Amar, M. (2008) Macroscopic lymph-node involvement and neck dissection predict lymphnode recurrence in papillary thyroid carcinoma. Eur $J$ Endocrinol 158, 551-560.

5. Haugen, B.R., Alexander, E.K., Bible, K.C., Doherty, G.M., Mandel, S.J., Nikiforov, Y.E., Pacini, F., Randolph, G.W., Sawka, A.M., Schlumberger, M., Schuff, K.G., Sherman, S.I., Sosa, J.A., Steward, D.L., Tuttle, R.M. \& Wartofsky, L. (2016) 2015 American Thyroid Association Management Guidelines for Adult Patients with Thyroid Nodules and Differentiated Thyroid Cancer: The American Thyroid Association Guidelines Task Force on Thyroid Nodules and Differentiated Thyroid Cancer. Thyroid 26, 1-133.

6. Vas Nunes, J.H., Clark, J.R., Gao, K., Chua, E., Campbell, P., Niles, N., Gargya, A. \& Elliott, M.S. (2013) Prognostic implications of lymph node yield and lymph node ratio in papillary thyroid carcinoma. Thyroid 23, 811-816.

7. Edge, S.B. \& Compton, C.C. (2010) The American Joint Committee on Cancer: the 7th edition of the AJCC cancer staging manual and the future of TNM. Ann Surg Oncol 17, 1471-1474.

8. Ito, Y., Kudo, T., Kobayashi, K., Miya, A., Ichihara, K. \& Miyauchi, A. (2012) Prognostic factors for recurrence of papillary thyroid carcinoma in the lymph nodes, lung, and bone: analysis of 5,768 patients with average 10-year follow-up. World J Surg 36, 1274-1278.

9. Su, D.H., Chang, S.H. \& Chang, T.C. (2015) The impact of locoregional recurrences and distant metastases on the survival of patients with papillary thyroid carcinoma. Clin Endocrinol (Oxf) 82, 286-294.

10. Schneider, D.F., Chen, H. \& Sippel, R.S. (2013) Impact of lymph node ratio on survival in papillary thyroid cancer. Ann Surg Oncol 20, 1906-1911.

11. Ito, Y., Kudo, T., Kihara, M., Takamura, Y., Kobayashi, K., Miya, A. \& Miyauchi, A. (2012) Prognosis of low-risk papillary thyroid carcinoma patients: its relationship with the size of primary tumors. Endocr J 59, 119-125.

12. Mazzaferri, E.L. (2007) Management of low-risk differentiated thyroid cancer. Endocr Pract 13, 498512.

13. Roti, E., degli Uberti, E.C., Bondanelli, M. \& Braverman, L.E. (2008) Thyroid papillary microcarcinoma: a descriptive and meta-analysis study. Eur J Endocrinol 159, 659-673.

14. Yuksel, U.M., Turanli, S., Acar, Y. \& Berberoglu, U. (2019) The prognostic factors for clinical N1b patients in thyroid papillary carcinoma. J Cancer Res Ther 15, 681-685.

15. Jesinghaus, M., Strehl, J., Boxberg, M., Bruhl, F., Wenzel, A., Konukiewitz, B., Schlitter, A.M., Steiger, K., Warth, A., Schnelzer, A., Kiechle, M., Beckmann, M.W., Noske, A., Hartmann, A., Mehlhorn, G., Koch, M.C. \& Weichert, W. (2018) Introducing a novel highly prognostic grading scheme based on tumour 
budding and cell nest size for squamous cell carcinoma of the uterine cervix. J Pathol Clin Res 4, 93102.

16. Weichert, W., Kossakowski, C., Harms, A., Schirmacher, P., Muley, T., Dienemann, H. \& Warth, A. (2016) Proposal of a prognostically relevant grading scheme for pulmonary squamous cell carcinoma. Eur Respir J 47, 938-946.

17. Zeng, R., Zhao, M., Niu, H., Yang, K.X., Shou, T., Zhang, G.Q. \& Yan, X.M. (2018) Relationship between Hashimoto's thyroiditis and papillary thyroid carcinoma in children and adolescents. Eur Rev Med Pharmacol Sci 22, 7778-7787.

18. Randolph, G.W., Duh, Q.Y., Heller, K.S., LiVolsi, V.A., Mandel, S.J., Steward, D.L., Tufano, R.P. \& Tuttle, R.M. (2012) The prognostic significance of nodal metastases from papillary thyroid carcinoma can be stratified based on the size and number of metastatic lymph nodes, as well as the presence of extranodal extension. Thyroid 22, 1144-1152.

19. Lee, K.E., Chung, I.Y., Kang, E., Koo do, H., Kim, K.H., Kim, S.W., Youn, Y.K. \& Oh, S.K. (2013) Ipsilateral and contralateral central lymph node metastasis in papillary thyroid cancer: patterns and predictive factors of nodal metastasis. Head Neck 35, 672-676.

20. Knezevic-Obad, A. \& Knezevic Stromar, I. (2008) [Cytomorphological characteristics of thyroid papillary carcinoma and their prognostic value]. Acta Med Croatica 62, 365-371.

21. The data that support the findings of this study are available on request from the corresponding author. The data are not publicly available due to privacy or ethical restrictions. 